\title{
Effect of the In-Plane Epitaxial Mismatch between the Substrate and the Film on the Properties of $\mathrm{YBa}_{2} \mathrm{Cu}_{3} \mathrm{O}_{7-\delta}$ Films
}

\author{
I.S. Abal'osheva, M.Z. Cieplak, Z. Adamus, M. Berkowski, \\ V. Domukhovski And M. Aleszkiewicz \\ Instytut Fizyki Polskiej Akademii Nauk \\ al. Lotników 32/46, 02-668 Warszawa, Poland
}

\begin{abstract}
We study the effect of the in-plane epitaxial mismatch between the substrate and the film on the crystallographic structure and the transport properties of $\mathrm{YBa}_{2} \mathrm{Cu}_{3} \mathrm{O}_{7-\delta}$ superconducting films of thicknesses ranging between 600 and $3000 \AA$. The films are grown by pulsed laser deposition on the new type of single-crystalline substrates prepared by Czochralski method, with the chemical formula $\left(\mathrm{SrAl}_{0.5} \mathrm{Ta}_{0.5} \mathrm{O}_{3}\right)_{0.7}\left(\mathrm{CaAl}_{0.5} \mathrm{Ta}_{0.5} \mathrm{O}_{3}\right)_{0.1}\left(\mathrm{LaAlO}_{3}\right)_{0.2}$. We find that superconducting properties of the samples are excellent, and generally they improve with increasing of the film thickness as a result of improved structural ordering. We also investigate the influence of the film thickness on the behavior of the critical current densities.
\end{abstract}

PACS numbers: 68.55.Jk, 74.25.Sv, 81.15.Aa

\section{Introduction}

The lattice mismatch between the substrate and the film is known to influence strongly the properties of thin films [1]. In order to produce reliable electronic devices on the base of the high- $T_{\mathrm{c}}$ superconductors (HTS) one must obtain high quality thin films with good transport properties. These properties are very sensitive to the conditions of the film growth, which may affect the microstructure and the strains built into the film. In HTS films strains commonly occur due to the mismatch between the crystal lattice parameters of films and substrates. The strains in the films usually relax via the formation of the arrays of so-called mismatch dislocations $[2,3]$, which are formed when the film thickness reaches its critical value [4]. In such a case the film thickness plays the role of parameter regulating strain distribution in films. The studies of the $\mathrm{YBa}_{2} \mathrm{Cu}_{3} \mathrm{O}_{7-\delta}$ (YBCO) films on commonly used substrates, $\mathrm{LaAlO}_{3}$ (LAO), $\mathrm{LaSrAlO}_{4}$ (LSAO), 
and $\mathrm{SrTiO}_{3}$ (STO) [5-8], indicate that the crystalline structure and the superconducting properties of $\mathrm{YBCO}$ are highly sensitive to the film thickness. The purpose of the present study is to evaluate the structural and superconducting properties of the films of different thicknesses ablated on the new type of substrate, $\left(\mathrm{SrAl}_{0.5} \mathrm{Ta}_{0.5} \mathrm{O}_{3}\right)_{0.7}\left(\mathrm{CaAl}_{0.5} \mathrm{Ta}_{0.5} \mathrm{O}_{3}\right)_{0.1}\left(\mathrm{LaAlO}_{3}\right)_{0.2}$ (SAT-CAT-LA), with the in-plane lattice parameter $a=3.8741 \AA$. Our study is the first attempt to examine in detail the properties of YBCO films grown on SAT-CAT-LA.

\section{Film preparation and measurement details}

Epitaxial $\mathrm{YBa}_{2} \mathrm{Cu}_{3} \mathrm{O}_{7-\delta}$ films were deposited by laser ablation on the $5 \times 5 \mathrm{~mm}^{2}$ SAT-CAT-LA substrates. Ablation was performed by $\mathrm{KrF}$ laser $(\lambda=248 \mathrm{~nm})$ with pulse duration $\tau=28 \mathrm{~ns}$, repetition rate -10 and $5 \mathrm{~Hz}$, energy density $-1.5 \mathrm{~J} / \mathrm{cm}^{2}$. The substrate temperature was held at $785^{\circ} \mathrm{C}$ and film preparation was carried out at $280 \mathrm{~m}$ Torr oxygen pressure, with subsequent cooling down to room temperature after deposition with the rate of $20 \mathrm{~K} / \mathrm{min}$ at $P_{\mathrm{O}_{2}}=300$ Torr. Thicknesses of the films were in the range between 60 and $300 \mathrm{~nm}$. Photolithography was used to pattern the films into $30 \mu \mathrm{m}-$ and $50 \mu \mathrm{m}$ strips for transport measurements.

The structural properties of the films were studied using a D-500 (Siemens) diffractometer and the film microstructure was investigated by atomic force microscope (AFM) Dimension 3100 Digital Instruments. The transport and superconducting properties of the films in the temperature range between 4.2 and $300 \mathrm{~K}$ were tested using the AC susceptibility measurements and the standard DC four-probe transport methods (resistance versus temperature, $R(T)$, and the currentvoltage $(I-V)$ characteristics).

\section{Experimental results and discussion}

The AFM images (Fig. 1a) show that the surface roughness of the films increases with the film thickness, $d$. This is a result of the replication and multiplication of surface irregularities and the change in the internal structure of the film. This is reflected in the magnitude of the root-mean-square roughness, defined as $R_{q}=\sqrt{\frac{1}{n} \sum_{n}\left(z_{i}-z_{\text {mean }}\right)^{2}}$ ( $n$ is the number of points on the surface), which increases as a function of $d$ (Fig. 1b).

The X-ray diffraction shows that all films grow with $c$ axis perpendicular to the substrate and to the film surface. Using the results of X-ray diffraction and AC susceptibility measurements, we have examined the correlations between various film parameters: the $c$-axis lattice parameter, the full width at half maximum (FWHM) of the rocking curve, the film thickness $d$, and the superconducting transition temperature, $T_{\mathrm{c}}$. We find that all films fall into two groups. The first group is characterized by a high value of FWHM, with a wide range of the $c$ parameter. The $T_{\mathrm{c}}$ of these films is seen to improve with increasing the value of $c$, 

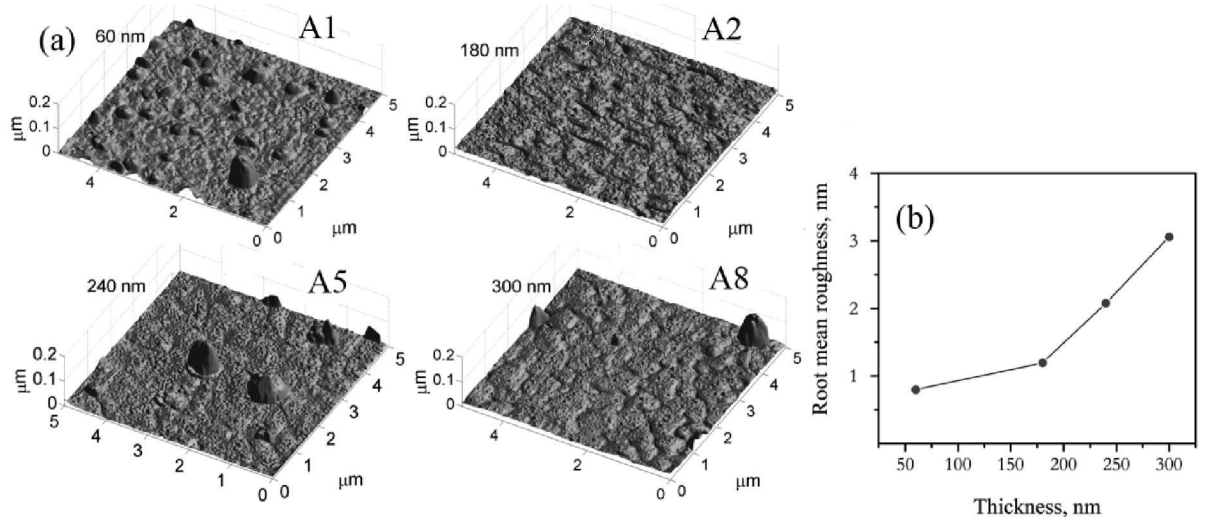

Fig. 1. (a) AFM images of several $\mathrm{YBa}_{2} \mathrm{Cu}_{3} \mathrm{O}_{7-\delta}$ films with different thicknesses. The labels 'Ax' are the film marking defined in Fig. 2. (b) The dependence of the root-mean-square roughness on the film thickness.

which is indicative of increasing in-plane compressive strain. The second group of films shows a lower value of FWHM, which suggests that these films grow more homogeneously. In these films the $T_{\mathrm{c}}$ is the largest for the $c$ value close to the bulk value and decreases with growing $c$. We believe that this may be a result of the stoichiometric deficiency caused by the interruption in ablation process due to laser instabilities.

The effect of the film thickness on the $c$ parameter is most obvious at small thicknesses, below $d \approx 150 \mathrm{~nm}$, where $c$ is decreasing rapidly with increasing $d$ until it reaches the value close to the bulk value. This is a result of the relief of the in-plane strain induced by the substrate on the film. However, some films of larger thicknesses exhibit increased $c$, presumably as a result of stoichiometric deficiencies. Interestingly, we find that the angle of the in-plane misalignment between the substrate and the film, $\Delta \varphi$, shows a much stronger and better defined correlation with the film thickness than the $c$ parameter. This dependence, shown in Fig. 2, indicates that the in-plane misalignment is small up to $d \approx 200-220 \mathrm{~nm}$. However for a larger $d$ the $\Delta \varphi$ abruptly increases up to $25-40 \%$. This is a clear

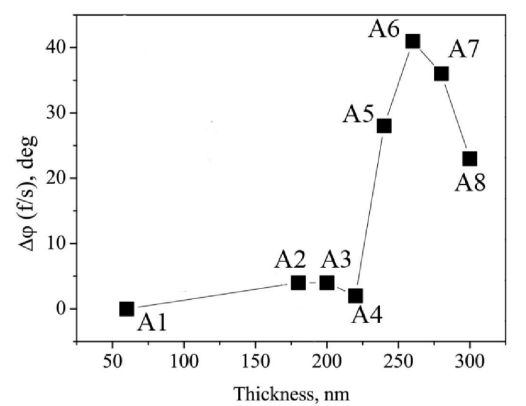

Fig. 2. The angle of $a b$-plane misalignment between the film and the substrate, $\Delta \varphi$, as a function of film thickness. 
evidence that $d \approx 200 \mathrm{~nm}$ is a limiting thickness above which the mismatch strain relaxation occurs.

We conclude that in the initial stage of the ablation process the film grows very flat (a small surface roughness) and well oriented with respect to the substrate. Film crystallographic cells are under the strain so that the value of $c$ parameter differs from the bulk. When the film thickness reaches the critical value of about 200-220 nm, the film microstructure abruptly changes: separate 3-dimensional (3D) grains grow strongly misoriented with respect to the substrate, the value of $c$ parameter becomes close to the bulk value, and the surface roughness increases.

These microstructural properties are reflected in the behavior of the critical current density, $J_{\mathrm{c}}$, as illustrated in Fig. 3 . The $J_{\mathrm{c}}$ grows with increasing $d$ reaching maximal values for $d \sim 200 \mathrm{~nm}$. A further increase in $d$ leads to the decrease in $J_{\mathrm{c}}$. A similar behavior has been observed previously in YBCO films $[7,8]$. The $J_{\mathrm{c}}$ is a linear function of temperatures at low $T$, as typically observed for YBCO thin films, and changes to nonlinear at higher $T$.

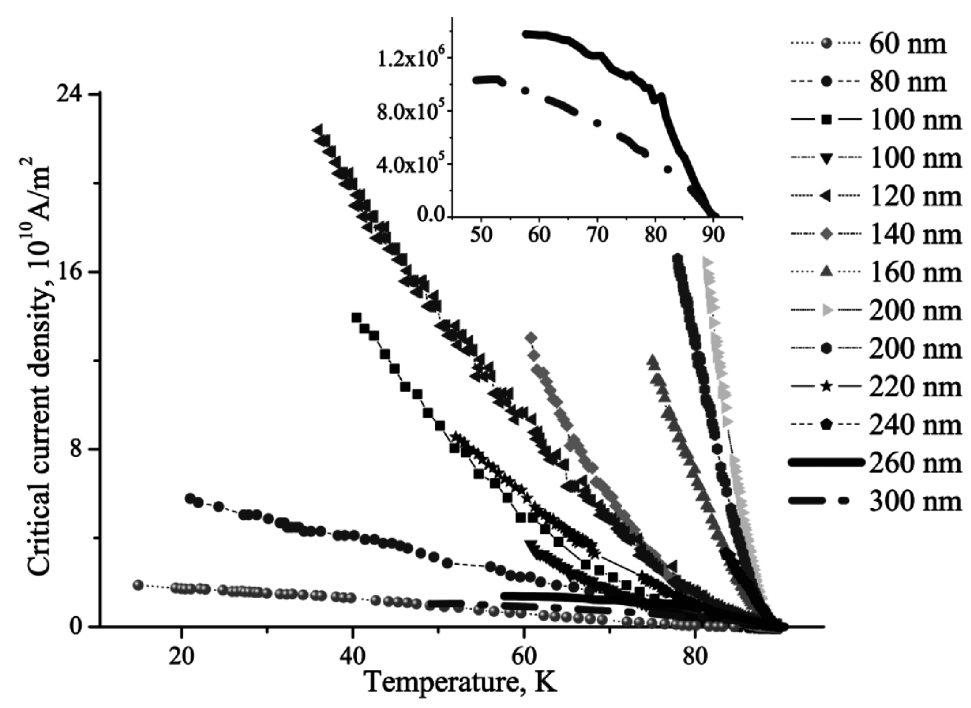

Fig. 3. The critical current density, $J_{\mathrm{c}}$, as a function of temperature for films with different thicknesses. Inset: a detail view of $J_{\mathrm{c}}(T)$ for films with thicknesses 260 and $300 \mathrm{~nm}$.

The growth of the $J_{\mathrm{c}}$ with $d$ up to $d \sim 200 \mathrm{~nm}$ may be explained by the increase of the amount of perfect superconducting $\mathrm{CuO}_{2}$ planes which take part in the current transport. At the small film thicknesses the transport is negatively affected by a "dead" layer, consisting of planes which are discontinuous somewhere on the path between the current leads as a result of the structural defects formed during the growth. With the increase in $d$ the amount of perfectly connected planes increases, contributing to the improvement of transport. This type of be- 
havior may be expected when the film grows in the mixed (Stranski-Kranstanov) growth mode. However, when the film thickness reaches the critical value of 200-220 nm, the further growth occurs in the form of misaligned grains as we infer from the structural results. The system of superconducting grains is likely to be coupled by Josephson junctions [9]. The behavior of the current in the grainboundary Josephson junctions inside a superconductor with $d_{x^{2}-y^{2} \text {-wave pairing }}$ symmetry depends on the angles $\alpha$ and $\beta$ formed between the crystalline axes on both sides of junction and the normal to the junction interface [10]. The dependence of the coefficient of the first term in the formula which describes the current in the junction, $I_{1}$, on the angles $\alpha$ and $\beta$, is given by the following expression [11]: $I_{1}=I_{S 1} \cos 2 \alpha \cos 2 \beta+I_{S 2} \sin 2 \alpha \sin 2 \beta$, so that, for example, $I_{1}$ reaches the maximum at $\alpha=\beta$, and $I_{1}=0$ when $\alpha=45^{\circ}$ and $\beta=0$. It is very likely that such a system of Josephson junctions is formed in thick laser-ablated YBCO films, resulting in a very large sensitivity of the critical current density to the misalignment of the grains.

\section{Conclusions}

The growth of YBCO thin films on the well-matched substrates of SAT-CAT-LA was investigated. It is found that the growth proceeds in two stages. In the first stage the film grows very well-oriented with respect to the substrate crystallographic axes, in the form of well-fused islands (the mixed growth mode). In this stage the films are strained despite the fact that SAT-CAT-LA substrate mismatch parameters are comparatively small, and the current-carrying capacities of film improve with the increasing thickness as a result of improved crystalline quality. In the second stage, when the film thickness reaches the critical value equal to $200-220 \mathrm{~nm}$, the film microstructure abruptly changes: separate 3D grains are strongly misoriented with respect to the substrate, and the critical current density decreases.

\section{Acknowledgments}

This work was supported by the State Committee for Scientific Research (Poland) under grant No. 2P 03B 04423.

\section{References}

[1] J.M. Philips, J. Appl. Phys. 79, 1829 (1996).

[2] E.A. Fitzgerald, Mater. Sci. Rep. 7, 87 (1991).

[3] T.J. Gosling, R. Bullough, S.C. Jain, J.R. Willis, J. Appl. Phys. 73, 8267 (1993).

[4] S.C. Jain, A.H. Harker, R.A. Cowley, Philos. Mag. A 75, 1461 (1997).

[5] C.B. Eom, A.F. Marshall, Y. Suzuki, T.H. Geballe, B. Boyer, R.F.W. Pease, R.B. van Dover, J.M. Phillips, Phys. Rev B. 48, 18 (1992).

[6] P. Zhang, T. Haage, A. Kazimirov, H.-U. Habermeier, T. Ruf, M. Cardona, J. Alloys Comp. 251, 70 (1997). 
[7] R.L.S. Emergo, J.Z. Wu, T. Aytug, D.K. Christen, Appl. Phys. Lett. 85, 618 (2002).

[8] B.W. Kang, A. Goyal, D.R. Lee, J.E. Mathis, E.D. Specht, P.M. Martin, D.M. Kroeger, M. Paranthaman, S. Sathyamurthy, J. Mater. Res. 17, 1750 (2002).

[9] M.S. Colclough, C.E. Keene, M. Gough, C.M. Muirhead, N. Thomas, J.S. Abell, S. Sutton, K.Y. Chen, B. Zhou, Nature 328, 47 (1987).

[10] Y. Tanaka, S. Kashiwaya, Phys. Rev. B 56, 892 (1997).

[11] M.B. Walker, J. Lüttmer-Strathmann, Phys. Rev. B 54, 588 (1996). 